hep-th/0106215

SNBNCBS-2001

\title{
Superfield approach to BRST cohomology
}

\author{
R. P. Malik 网 \\ S. N. Bose National Centre for Basic Sciences, \\ Block-JD, Sector-III, Salt Lake, Calcutta- 700 098, India
}

\begin{abstract}
In the framework of superfield formalism, we discuss some aspects of the cohomological features of a two $(1+1)$-dimensional free Abelian gauge theory described by a Becchi-Rouet-Stora-Tyutin (BRST) invariant Lagrangian density. We demonstrate that the conserved and nilpotent (anti-)BRST- and (anti-)co-BRST charges are the generators of translations along the Grassmannian directions of the four (2+2)-dimensional supermanifold. A bosonic symmetry is shown to be generated by a Noether conserved charge that generates a translation along a bosonic direction of the supermanifold which turns out to be equivalent to a couple of successive translations along the two different and independent Grassmannian directions of the same supermanifold. Algebraically, these charges are found to be analogous to the de Rham cohomology operators of differential geometry.
\end{abstract}

\footnotetext{
* E-mail address: malik@boson.bose.res.in
} 


\section{Introduction}

The existence of some new local, covariant and continuous symmetries and their possible connections with the mathematics of differential geometry, cohomology and Hodge decomposition theorem (HDT) for the two $(1+1)$-dimensional $(2 \mathrm{D})$ free- as well as interacting (non-)Abelian gauge theories have been recently established in a set of papers [1-5]. The topological nature of the 2D free Abelian- and self-interacting non-Abelian gauge theories (having no interaction with matter fields) has also been demonstrated by exploiting the conserved and nilpotent (anti-)BRST charges $\left(Q_{(a) b}\right)$, (anti-)dual BRST charges $\left(Q_{(a) d}\right)$, a conserved ghost charge $Q_{g}$ and a bosonic charge $Q_{w}=\left\{Q_{d}, Q_{b}\right\}=\left\{Q_{a b}, Q_{a d}\right\}$ which generate some interesting local, covariant and continuous symmetry transformations for the 2D BRST invariant Lagrangian density [6]. These local conserved charges have also been utilized to express HDT in the quantum Hilbert space of states where any arbitrary state $\mid \Psi>_{n}$ with the ghost number $n$, (i.e. $i Q_{g}\left|\Psi>_{n}=n\right| \Psi>_{n}$ ) can be written as the sum of a harmonic state $\mid \omega>_{n}\left(Q_{w}\left|\omega>_{n}=0, Q_{b}\right| \omega>_{n}=0, Q_{d} \mid \omega>_{n}=0\right)$, a BRST exact state $Q_{b} \mid \xi>_{n-1}$ and a co-BRST exact state $Q_{d} \mid \chi>_{n+1}$ as

$$
\left|\Psi>_{n}=\right| \omega>_{n}+Q_{b}\left|\xi>_{n-1}+Q_{d}\right| \chi>_{n+1} \equiv\left|\omega>_{n}+Q_{a d}\right| \xi>_{n-1}+Q_{a b} \mid \chi>_{n+1} .
$$

The above equation encodes the fact that, in the BRST formalism, there are two sets of conserved charges (viz. $\left(Q_{b}, Q_{d}, Q_{w}\right)$ and $\left.\left(Q_{a d}, Q_{a b}, Q_{w}\right)\right)$ that are analogous to the de Rham cohomology operators of differential geometry. The origin for the existence of this mapping: $\left(Q_{b}, Q_{a d}\right) \Leftrightarrow d,\left(Q_{d}, Q_{a b}\right) \Leftrightarrow \delta, Q_{w}=\left\{Q_{(a) b}, Q_{(a) d}\right\} \Leftrightarrow \Delta$ has not been made clear in our earlier works [1-6]. In the present paper, we clarify the existence of such a mapping.

In the physical four $(3+1)$-dimensional $(4 \mathrm{D})$ flat Minkowskian spacetime, the above type of symmetries and corresponding local and conserved charges have been exploited for the definition of the celebrated HDT in the context of free Abelian two-form gauge theory [11]. Furthermore, these local and conserved charges have been shown to obey exactly the same kind of algebra as that of the de Rham cohomology operators of differential geometry defined on a compact manifold $[1,5,6,11]$. The existence of some subtle discrete symmetries has been shown to correspond to the Hodge $*$ duality operation of the ordinary differential geometry for the case of 2D one-form- and 4D two-form Abelian gauge theories $[6,11]$. Under these discrete symmetries, the (anti-)BRST- and (anti-)co-BRST symmetry transformations are related to one-another in exactly the same way as the cohomological operators $d$ and $\delta= \pm * d *$ are related to each-other [6]. In our present paper, we show that a proper generalization of these discrete symmetries for the case of a $2 \mathrm{D}$ free $U(1)$ gauge

${ }^{\dagger}$ This equation is the analogue of the HDT which states that any arbitrary $n$-form $f_{n}(n=0,1,2, \ldots)$, on a compact manifold, can be decomposed into a harmonic form $\omega_{n}\left(\Delta \omega_{n}=0, d \omega_{n}=0, \delta \omega_{n}=0\right)$, an exact form $d g_{n-1}$ and a co-exact form $\delta h_{n+1}$ as: $f_{n}=\omega_{n}+d g_{n-1}+\delta h_{n+1}$ where $\delta(= \pm * d *)$ is the Hodge dual of $d$ (with $d^{2}=0, \delta^{2}=0 ; *=$ Hodge duality operation) and Laplacian $\Delta$ is defined as $\Delta=(d+\delta)^{2}=d \delta+\delta d[7-10]$. The set $(d, \delta, \Delta)$ is known as the de Rham cohomology operators of differential geometry and they obey the algebra: $d^{2}=\delta^{2}=0,[\Delta, d]=[\Delta, \delta]=0, \Delta=\{d, \delta\}=(d+\delta)^{2}$. 
theory in the superfield formulation (together with the idea of Hodge decomposition for the 2D fermionic vector fields) provides a logical origin for the existence of the "off-shell" nilpotent, local, covariant and continuous (anti-)BRST- and (anti-)co-BRST symmetries. The "on-shell" version of these results has already been obtained in Ref. [12]. We lay emphasis on the fact that, in our present attempt, we concentrate only on the "off-shell" version of these nilpotent symmetries which are more general than their "on-shell" counterparts. It is evident that we do not exploit the classical equations of motion anywhere in our present discussion for the off-shell nilpotent (anti-)BRST- and (anti-)co-BRST symmetries.

One of the most popular geometrical approaches to the BRST formalism (in the language of the Maurer-Cartan equation and the translation generators) is the superfield formulation [13-18]. In this superfield formulation, so far, only the mathematical power of the super exterior derivative $\tilde{d}$, together with the idea of the horizontality condition $f$ on the two-form constructed with it, has been tapped which leads to the interpretation of the nilpotent (anti-)BRST charges as the generators for translations along the Grassmannian directions of the supermanifold for the one-form gauge theory [13-15]. In view of the generality and applicability of this formulation to any arbitrary $n$-form $(n=1,2 \ldots)$ gauge theories in any arbitrary spacetime dimension [15-18], it is an interesting endeavour to provide the geometrical interpretation for the nilpotent (anti-)dual BRST symmetries and a bosonic symmetry (generated by the Casimir operator) [1-6,11] in the language of other super de Rham cohomology operators $\tilde{\delta}= \pm \star \tilde{d} \star$ and $\tilde{\Delta}=\tilde{d} \tilde{\delta}+\tilde{\delta} \tilde{d}$ defined on the four $(2+2)$-dimensional compact supermanifold. Here $\tilde{\delta}$ and $\tilde{\Delta}$ are the super co-exterior derivative and super Laplacian operator, respectively. To the best of our knowledge, these super cohomological operators have not yet been exploited in the context of superfield approach to BRST formalism, BRST cohomology and HDT in the quantum Hilbert space of states for any gauge theory in any spacetime dimension. One of the central themes of the present paper is to make an attempt towards this goal and to show that useful symmetries do emerge for the $2 D$ free Abelian gauge theory when we exploit these geometrical (cohomological) super operators together with the idea of the generalized version of horizontality condition. We demonstrate that the off-shell nilpotent (anti-)dual BRST symmetries (generated by (anti-)co-BRST charges) emerge when we exploit the super co-exterior derivative $\tilde{\delta}= \pm \star \tilde{d} \star$ together with an analogue of the "horizontality" condition. Geometrically, the nilpotent and conserved (anti-)co-BRST charges (similar to their counterpart (anti-)BRST charges [15]) turn out to be the translation generators along the Grassmannian directions 3 of the supermanifold. When we exploit the mathematical power of super Laplacian operator $\tilde{\Delta}=\tilde{d} \tilde{\delta}+\tilde{\delta} \tilde{d}$ in this context, we obtain a bosonic symmetry transformation for the $U(1)$ gauge field alone and ghost fields do not transform at all. As expected, the Noether

\footnotetext{
$\ddagger$ This condition is referred to as the "soul flatness" in Ref. [18] implying the flatness of the curvature (two-form) tensor in the Grassmannian directions of the supermanifold.

$\S$ Even though (anti-)BRST- and (anti-)co-BRST charges generate translations along the Grassmannian directions, there is a clear distinction between them. To be precise, these charges generate translations for the fermionic (anti-)ghost fields along different Grassmannian directions of the supermanifold (cf.Sec.4).
} 
conserved charge corresponding to this symmetry emerges as the generator for the above symmetry transformations. In the language of the geometry on the supermanifold, this bosonic charge (Casimir operator) turns out to be the generator of translations along the $\theta \bar{\theta}$-direction of the supermanifold. In fact, this bosonic direction is equivalent to a couple of successive translations along the Grassmannian $(\theta$ and $\bar{\theta})$ directions of the supermanifold.

This paper is organized as follows. In section 2, we set up the notations and recapitulate the bare essentials of our earlier works [1-6] for the 2D free Abelian gauge theory in the framework of Lagrangian formulation. This is followed by the derivation of the (anti-)BRST symmetries in the framework of superfield formalism in section 3 by exploiting the idea of "horizontality" condition [15]. In section 4, we derive the (anti-)dual BRST symmetries by exploiting the super dual exterior derivative $\tilde{\delta}$ together with a restriction $(\tilde{\delta} \tilde{A}=\delta A)$ which is the analogue of the horizontality condition. Section 5 is devoted to the discussion of some new discrete symmetries that are present in the superfield formulation. In section 6 , we derive a bosonic symmetry by exploiting the mathematical power of $\tilde{\Delta}$ together with a "horizontality type" restriction w.r.t. this operator. Finally, in section 7, we make some concluding remarks and point out some future directions that can be pursued later.

\section{Preliminary: (anti-)BRST- and (anti-)co-BRST symmetries}

We begin with the BRST invariant Lagrangian density $\left(\mathcal{L}_{b}\right)$ for the flat Minkowskian two $(1+1)$-dimensional [1] free Abelian gauge theory in the Feynman gauge (see, e.g., [18-20])

$$
\mathcal{L}_{b}=-\frac{1}{4} F^{\mu \nu} F_{\mu \nu}-\frac{1}{2}(\partial \cdot A)^{2}-i \partial_{\mu} \bar{C} \partial^{\mu} C \equiv \frac{1}{2} E^{2}-\frac{1}{2}(\partial \cdot A)^{2}-i \partial_{\mu} \bar{C} \partial^{\mu} C
$$

where $F_{\mu \nu}=\partial_{\mu} A_{\nu}-\partial_{\nu} A_{\mu}$ (with $F_{01}=E$ as electric field) is the field strength tensor derived from the one-form $A=d x^{\mu} A_{\mu}$ (with $A_{\mu}$ as the vector potential) by application of the exterior derivative $d$ (i.e. $F=d A$ ). On the other hand, the gauge-fixing term $(\partial \cdot A)$ is derived from the one-form $A=d x^{\mu} A_{\mu}$ by application of the co-exterior derivative $\delta$ (i.e. $(\partial \cdot A)=\delta A, \delta=-* d *)$. Thus, two-form $F=d A$ and zero-form $(\partial \cdot A)=\delta A$ are 'Hodge' dual to each-other. The (anti-)ghost fields $(\bar{C}) C$ are anti-commuting $\left(\bar{C}^{2}=C^{2}=0, C \bar{C}=\right.$ $-\bar{C} C)$ in nature. One can linearize the kinetic energy term and the gauge-fixing term by introducing auxiliary-fields $\mathcal{B}$ and $B$, as

$$
\mathcal{L}_{B}=\mathcal{B} E-\frac{1}{2} \mathcal{B}^{2}+B(\partial \cdot A)+\frac{1}{2} B^{2}-i \partial_{\mu} \bar{C} \partial^{\mu} C
$$

This Lagrangian density respects the following off-shell nilpotent $\left(s_{b}^{2}=0, s_{d}^{2}=0\right)$ BRST $\left(s_{b}\right)$ and dual-BRST $\left(s_{d}\right)$ symmetry transformations !

$$
\begin{aligned}
& s_{b} A_{\mu}=\partial_{\mu} C \quad s_{b} C=0 \quad s_{b} \mathcal{B}=0 \quad s_{b} E=0 \\
& s_{b} \bar{C}=i B \quad s_{b} B=0 \quad s_{b}(\partial \cdot A)=\square C
\end{aligned}
$$

I We adopt here the notations and conventions in which the 2D flat Minkowski metric is : $\eta_{\mu \nu}=\operatorname{diag}$ $(+1,-1)$ and $\square=\eta^{\mu \nu} \partial_{\mu} \partial_{\nu}=\partial_{0} \partial_{0}-\partial_{1} \partial_{1}, F_{01}=-\varepsilon^{\mu \nu} \partial_{\mu} A_{\nu}=\partial_{0} A_{1}-\partial_{1} A_{0}=E=F^{10}, \varepsilon_{01}=\varepsilon^{10}=+1$.

$\|$ We follow here the notations adopted in Ref. [21]. In fact, a BRST transformation $\delta_{B}$ is the product of an anti-commuting constant parameter $\zeta$ and the transformation $s_{b}$ (i.e. $\delta_{B}=\zeta s_{b}$ ) in its full glory. 


$$
\begin{array}{llll}
s_{d} A_{\mu} & =-\varepsilon_{\mu \nu} \partial^{\nu} \bar{C} & s_{d} C=-i \mathcal{B} & s_{d} \mathcal{B}=0 \\
s_{d} E & =\square \bar{C} \quad s_{d} \bar{C}=0 & s_{d} B=0 & s_{d}(\partial \cdot A)=0 .
\end{array}
$$

The corresponding off-shell nilpotent $\left(s_{a b}^{2}=0, s_{a d}^{2}=0\right)$ anti-BRST $\left(s_{a b}\right)$ symmetries (with $\left.s_{b} s_{a b}+s_{a b} s_{b}=0\right)$ and anti-co-BRST $\left(s_{a d}\right)$ symmetries (with $s_{d} s_{a d}+s_{a d} s_{d}=0$ ) are

$$
\begin{aligned}
& s_{a b} A_{\mu}=\partial_{\mu} \bar{C} \quad s_{a b} \bar{C}=0 \quad s_{a b} \mathcal{B}=0 \quad s_{a b} E=0 \\
& s_{a b} C=-i B \quad s_{a b} B=0 \quad s_{a b}(\partial \cdot A)=\square \bar{C} \\
& s_{a d} A_{\mu}=-\varepsilon_{\mu \nu} \partial^{\nu} C \quad s_{a d} \bar{C}=i \mathcal{B} \quad s_{a d} \mathcal{B}=0 \\
& s_{a d} E \quad=\square C \quad s_{a d} C=0 \quad s_{a d} B=0 \quad s_{a d}(\partial \cdot A)=0 .
\end{aligned}
$$

The anti-commutators of the above symmetries lead to the definition of a bosonic symmetry $s_{w}=\left\{s_{b}, s_{d}\right\}=\left\{s_{a b}, s_{a d}\right\}, s_{w}^{2} \neq 0$. Under this symmetry, the (anti-)ghost fields do not transform and only the $U(1)$ gauge field transform as $[1,2,6]$

$$
\begin{aligned}
& s_{w} A_{\mu}=\left(\partial_{\mu} \mathcal{B}+\varepsilon_{\mu \nu} \partial^{\nu} B\right) \equiv \varepsilon_{\mu \nu}\left(\partial^{\nu} B+\varepsilon^{\nu \lambda} \partial_{\lambda} \mathcal{B}\right) \quad s_{w} B=0 \\
& s_{w} C=0 \quad s_{w} \bar{C}=0 \quad s_{w} \mathcal{B}=0 \quad s_{w}(\partial \cdot A)=\square \mathcal{B} \quad s_{w} E=-\square B .
\end{aligned}
$$

It can be seen that the transformation for the $U(1)$ gauge field $A_{\mu}$ is just equal to its own equation of motion: $\partial_{\mu} \mathcal{B}+\varepsilon_{\mu \nu} \partial^{\nu} B(=0)$. The substitutions $\mathcal{B}=E, B=-(\partial \cdot A)$ make the above equation of motion equal to $-\varepsilon_{\mu \nu} \square A^{\nu}(=0)$. In terms of the vector fields $A_{\mu}$ alone, now we have the transformation $\tilde{s}_{w} A_{\mu}=-\varepsilon_{\mu \nu} \square A^{\nu}$. Thus, we note that all the transformations $s_{w}$ in (2.7) (and their analogues $\tilde{s}_{w}$ ) are trivially zero on the on-shell **. All the above continuous symmetry transformations can be concisely expressed, in terms of generators $Q_{r}$ (see, e.g., Sec. 7 for explicit local expressions) and generic field $\phi$, as

$$
s_{r} \phi=-i\left[\phi, Q_{r}\right]_{ \pm} \quad r=b, a b, d, a d, w
$$

where $Q_{r}$ are the conserved Noether charges derived from the conserved currents and brackets $[,]_{ \pm}$stand for the (anti-)commutators for $\phi$ being (fermionic)bosonic in nature.

Now we shall dwell a bit on the presence of some interesting discrete symmetries in the theory. It can be seen from (2.3) and (2.5) that $s_{b} \leftrightarrow s_{a b}$ for the discrete transformations: $C \leftrightarrow \bar{C}, B \leftrightarrow-B$. In a similar fashion, it is clear from (2.4) and (2.6) that $s_{d} \leftrightarrow s_{a d}$ for the presence of the discrete transformations: $C \leftrightarrow \bar{C}, \mathcal{B} \leftrightarrow-\mathcal{B}$. In other words, the discrete symmetries: $C \leftrightarrow \bar{C}, B \leftrightarrow-B, \mathcal{B} \leftrightarrow-\mathcal{B}, A_{\mu} \leftrightarrow A_{\mu}$, connect the BRST to anti-BRST and the co-BRST to anti-co-BRST symmetries and vice-versa. Yet another interesting discrete symmetry transformations $[1,2,6]$

$$
\begin{array}{lllc}
C \rightarrow \pm i \bar{C} & E \rightarrow \pm i(\partial \cdot A) & A_{\mu} \rightarrow A_{\mu} & \partial_{\mu} \rightarrow \pm i \varepsilon_{\mu \nu} \partial^{\nu} \\
\bar{C} \rightarrow \pm i C & (\partial \cdot A) \rightarrow \pm i E & \mathcal{B} \rightarrow \mp i B & B \rightarrow \mp i \mathcal{B}
\end{array}
$$

connect $s_{b}$ with $s_{d}$ (as well as $s_{a b}$ with $s_{a d}$ ) and leave the Lagrangian density (2.2) forminvariant [6]. The topological nature of this theory has been shown by demonstrating that

** We shall be exploiting this observation in Secs. 6 and 7. It will be noticed, however, that $\tilde{s}_{w}$ (with $\left.\tilde{s}_{w} C=0, \tilde{s}_{w} \bar{C}=0, \tilde{s}_{w} A_{\mu}=-\varepsilon_{\mu \nu} \square A^{\nu}\right)$ is a symmetry transformation for the Lagrangian density (2.1) and is the analogue of the symmetry $s_{w}$ (cf.(2.7)) for the Lagrangian density (2.2). 
the Lagrangian density in (2.1) can be written, modulo some total derivatives, as the sum of BRST- and dual BRST anti-commutators $[1,2,6]$

$$
\begin{aligned}
& \mathcal{L}_{b}=\left\{Q_{d}, T_{1}\right\}+\left\{Q_{b}, T_{2}\right\} \equiv s_{d}\left(i T_{1}\right)+s_{b}\left(i T_{2}\right) \\
& \mathcal{L}_{b}=\left\{Q_{a d}, P_{1}\right\}+\left\{Q_{a b}, P_{2}\right\} \equiv s_{a d}\left(i P_{1}\right)+s_{a b}\left(i P_{2}\right)
\end{aligned}
$$

where the local expressions for $T_{(1,2)}$ and $P_{(1,2)}$ are: $T_{1}=\frac{1}{2} E C, T_{2}=-\frac{1}{2}(\partial \cdot A) \bar{C}, P_{1}=$ $-\frac{1}{2} E \bar{C}, P_{2}=\frac{1}{2}(\partial \cdot A) C$. Similarly, the off-shell nilpotent (anti-)BRST- and (anti-)dual BRST invariant Lagrangian density (2.2) can be written in two different ways as: (i) the sum of the kinetic energy term and an (anti-)BRST invariant part or (ii) the sum of the gauge-fixing term and an (anti-)dual BRST invariant part. These alternative ways of expressing the Lagrangian density are

$$
\mathcal{L}_{B}=\mathcal{B} E-\frac{1}{2} \mathcal{B}^{2}+s_{b} s_{a b}\left(\frac{i}{2} A^{2}-\frac{1}{2} \bar{C} C\right) \equiv B(\partial \cdot A)+\frac{1}{2} B^{2}+s_{d} s_{a d}\left(\frac{i}{2} A^{2}-\frac{1}{2} \bar{C} C\right) .
$$

The topological invariants for this theory and their well-known recursion relations have been computed on a 2D compact manifold $[1,2,6]$. Energy-momentum tensor has been shown to be the sum of BRST- and co-BRST anti-commutators [2,6].

\section{Superfield formulation for (anti-)BRST symmetry transformations}

We start off with a four $(2+2)$ dimensional supermanifold that is parametrized by two c-number commuting (bosonic) spacetime coordinates $x^{\mu}(\mu=0,1)$ and two Grassmann variables $\theta$ and $\bar{\theta}$. We define a supervector superfield $v_{s}$ on this supermanifold as [15]

$$
v_{s}=\left(\Phi_{\mu}(x, \theta, \bar{\theta}), \eta(x, \theta, \bar{\theta}), \bar{\eta}(x, \theta, \bar{\theta})\right)
$$

where $\Phi_{\mu}(x, \theta, \bar{\theta})$ are the bosonic (even) superfields and $\eta(x, \theta, \bar{\theta}), \bar{\eta}(x, \theta, \bar{\theta})$ are the fermionic (odd) superfields which constitute the supermultiplet of the supervector superfield $v_{s}$. In terms of the superspace coordinates $x^{\mu}, \theta, \bar{\theta}$, the component superfields can be expanded as

$$
\begin{aligned}
\Phi_{\mu}(x, \theta, \bar{\theta}) & =A_{\mu}(x)+\theta \bar{R}_{\mu}(x)+\bar{\theta} R_{\mu}(x)+i \theta \bar{\theta} S_{\mu}(x) \\
\eta(x, \theta, \bar{\theta}) & =C(x)+i \theta \bar{B}(x)+i \bar{\theta} \mathcal{B}(x)+i \theta \bar{\theta} s(x) \\
\bar{\eta}(x, \theta, \bar{\theta}) & =\bar{C}(x)+i \theta \overline{\mathcal{B}}(x)+i \bar{\theta} B(x)+i \theta \bar{\theta} \bar{s}(x)
\end{aligned}
$$

where field variables $A_{\mu}(x) \equiv A_{\mu}(x, 0,0) ; C(x) \equiv C(x, 0,0)$, etc. are functions of only spacetime variables. It will be noticed that the local fields: $A_{\mu}(x), S_{\mu}(x), B(x), \bar{B}(x), \mathcal{B}(x), \overline{\mathcal{B}}(x)$ are bosonic (even) in nature whereas the local fermionic (odd) fields in the theory are: $R_{\mu}(x), \bar{R}_{\mu}(x), C(x), \bar{C}(x), s(x), \bar{s}(x)$. The super exterior derivative $\tilde{d}=d z^{M} \partial_{M}$ on this supermanifold is defined in terms of the superspace differentials $\left(d x^{\mu}, d \theta, d \bar{\theta}\right)$ as $[22]$

$$
\tilde{d}=d x^{\mu} \partial_{\mu}+d \theta \partial_{\theta}+d \bar{\theta} \partial_{\bar{\theta}}
$$

${ }^{\dagger \dagger}$ We follow the field notations of Ref. [13,15,17] but be consistent with the conventions adopted in Ref. [22] for the definition of the super derivatives and super differential forms on a compact supermanifold. 
where

$$
\begin{array}{lll}
\tilde{d}=d z^{M} \frac{\partial}{\partial z^{M}} \quad \tilde{d}^{2}=0 & z^{M}=\left(x^{\mu}, \theta, \bar{\theta}\right) \\
\partial_{\mu}=\frac{\partial}{\partial x^{\mu}} \quad \partial_{\theta}=\frac{\partial}{\partial \theta} & \partial_{\bar{\theta}}=\frac{\partial}{\partial \bar{\theta}} .
\end{array}
$$

A connection super one-form on the supermanifold can be defined in terms of the component superfields of the supervector superfield $v_{s}$ as

$$
\tilde{A}=d x^{\mu} \Phi_{\mu}(x, \theta, \bar{\theta})+d \theta \bar{\eta}(x, \theta, \bar{\theta})+d \bar{\theta} \eta(x, \theta, \bar{\theta}) .
$$

The Maurer-Cartan equation that defines the curvature two-form $\tilde{F}$ from the connection one-form $\tilde{A}$ and the exterior derivative $\tilde{d}$ on the supermanifold, in its most general form, is

$$
\tilde{F}=\tilde{d} \tilde{A}+\tilde{A} \wedge \tilde{A} \equiv \tilde{d} \tilde{A}+\frac{1}{2}[\tilde{A}, \tilde{A}] .
$$

For Abelian $U(1)$ gauge theory, however, the last term in the above equation is zero (i.e., $\tilde{A} \wedge$ $\left.\tilde{A}=\frac{1}{2}[\tilde{A}, \tilde{A}]=0\right)$. The horizontality condition imposes the restriction that the components of the curvature two-form $\tilde{F}=\tilde{d} \tilde{A}$ for the Abelian gauge theory (with $\{d \theta(d \bar{\theta}), \theta(\bar{\theta}\}=0$ )

$$
\begin{aligned}
\tilde{F}=\tilde{d} \tilde{A} & =\left(d x^{\mu} \wedge d x^{\nu}\right)\left(\partial_{\mu} \Phi_{\nu}\right)+\left(d x^{\mu} \wedge d \theta\right)\left(\partial_{\mu} \bar{\eta}-\partial_{\theta} \Phi_{\mu}\right)-(d \theta \wedge d \theta)\left(\partial_{\theta} \bar{\eta}\right) \\
& +\left(d x^{\mu} \wedge d \bar{\theta}\right)\left(\partial_{\mu} \eta-\partial_{\bar{\theta}} \Phi_{\mu}\right)-(d \theta \wedge d \bar{\theta})\left(\partial_{\theta} \bar{\eta}+\partial_{\bar{\theta}} \eta\right)-(d \bar{\theta} \wedge d \bar{\theta})\left(\partial_{\bar{\theta}} \eta\right)
\end{aligned}
$$

must vanish along the Grassmannian directions $(\theta, \bar{\theta})$ of the supermanifold. This ultimately amounts to the following condition (which is nothing but $\tilde{d} \tilde{A}=d A$ )

$$
\tilde{F} \equiv \frac{1}{2}\left(d z^{M} \wedge d z^{N}\right) \tilde{F}_{M N}=\frac{1}{2}\left(d x^{\mu} \wedge d x^{\nu}\right) F_{\mu \nu} \equiv F
$$

where the wedge product $d z^{M} \wedge d z^{N}$, in the component form of the superspace variables, is: $d x^{\mu} \wedge d x^{\nu}=-d x^{\nu} \wedge d x^{\mu}, d \theta \wedge d \bar{\theta}=d \bar{\theta} \wedge d \theta, d x^{\mu} \wedge d \theta=-d \theta \wedge d x^{\mu}$, etc. In the following, the above horizontality condition (3.7) leads to

$$
\begin{array}{lll}
\partial_{\theta} \bar{\eta}=0 & \rightarrow \overline{\mathcal{B}}=0 \quad \bar{s}=0 \\
\partial_{\bar{\theta}} \eta=0 & \rightarrow \mathcal{B}=0 \quad s=0 \\
\partial_{\theta} \eta=-\partial_{\bar{\theta}} \bar{\eta} & \rightarrow B+\bar{B}=0 \\
\partial_{\mu} \eta=\partial_{\bar{\theta}} \Phi_{\mu} & \rightarrow R_{\mu}=\partial_{\mu} C \quad S_{\mu}=-\partial_{\mu} \bar{B} \equiv \partial_{\mu} B \\
\partial_{\mu} \bar{\eta}=\partial_{\theta} \Phi_{\mu} & \rightarrow \quad \bar{R}_{\mu}=\partial_{\mu} \bar{C} \quad S_{\mu}=\partial_{\mu} B
\end{array}
$$

which satisfy the other conditions, namely;

$$
\partial_{\mu} \bar{R}_{\nu}-\partial_{\nu} \bar{R}_{\mu}=0 \quad \partial_{\mu} R_{\nu}-\partial_{\nu} R_{\mu}=0 \quad \partial_{\mu} S_{\nu}-\partial_{\nu} S_{\mu}=0 .
$$

It is clear (cf.Sec.2) that the (anti-)BRST charges are the generators (cf.(2.8)) of translations along the Grassmannian directions (i.e., $\operatorname{Lim}_{\theta, \bar{\theta} \rightarrow 0} \frac{\partial}{\partial \theta(\theta)} \Sigma(x, \theta, \bar{\theta})=i\left[Q_{(a) b}, \Lambda\right]_{ \pm}$, for $\Sigma=$ $\eta, \bar{\eta}, \Phi_{\mu}$ and corresponding $\left.\Lambda=C, \bar{C}, A_{\mu}\right)$ of the supermanifold as the expansions in (3.2) can be recast in terms of $s_{(a) b}$ as

$$
\begin{aligned}
\Phi_{\mu}(x, \theta, \bar{\theta}) & =A_{\mu}(x)+\theta\left(s_{a b} A_{\mu}(x)\right)+\bar{\theta}\left(s_{b} A_{\mu}(x)\right)+\theta \bar{\theta}\left(s_{b} s_{a b} A_{\mu}(x)\right) \\
\eta(x, \theta, \bar{\theta}) & =C(x)+\theta\left(s_{a b} C(x)\right)+\bar{\theta}\left(s_{b} C(x)\right)+\theta \bar{\theta}\left(s_{b} s_{a b} C(x)\right) \\
\bar{\eta}(x, \theta, \bar{\theta}) & =\bar{C}(x)+\theta\left(s_{a b} \bar{C}(x)\right)+\bar{\theta}\left(s_{b} \bar{C}(x)\right)+\theta \bar{\theta}\left(s_{b} s_{a b} \bar{C}(x)\right)
\end{aligned}
$$


which finally amounts to the following expansion

$$
\begin{aligned}
\Phi_{\mu}(x, \theta, \bar{\theta}) & =A_{\mu}(x)+\theta \partial_{\mu} \bar{C}(x)+\bar{\theta} \partial_{\mu} C(x)+i \theta \bar{\theta} \partial_{\mu} B(x) \\
\eta(x, \theta, \bar{\theta}) & =C(x)+i \theta \bar{B}(x) \equiv C(x)-i \theta B(x) \\
\bar{\eta}(x, \theta, \bar{\theta}) & =\bar{C}(x)+i \bar{\theta} B(x) .
\end{aligned}
$$

This, in a nut-shell, provides the origin for the existence of (anti-)BRST symmetries in the framework of superfield formulation.

\section{Superfield approach to (anti-)dual BRST transformations}

On an even dimensional ordinary spacetime manifold, it is obvious that the operation of $\delta=-* d *$ on the one-form $A=d x^{\mu} A_{\mu}$ leads to the zero-form as none other than the gauge-fixing term $(\partial \cdot A)[7,8]$ :

$$
\delta A=-* d * A=(\partial \cdot A)
$$

where the Hodge $*$ duality operation on the ordinary differentials in the flat Minkowskian two-dimensional spacetime is: $*\left(d x^{\mu}\right)=\varepsilon^{\mu \nu}\left(d x_{\nu}\right), *\left(d x^{\mu} \wedge d x^{\nu}\right)=\varepsilon^{\mu \nu}$. Now, on the connection super one-form $\tilde{A}$ defined on a $2+2$-dimensional supermanifold, we apply the super co-exterior derivative $\tilde{\delta}$ and exploit the following condition

$$
\tilde{\delta} \tilde{A}=-\star \tilde{d} \star \tilde{A}=(\partial \cdot A) .
$$

In words, this equation amounts to restricting the zero-form superscalar superfield $(\tilde{\delta} \tilde{A})$, that emerges after the application of $\tilde{\delta}$ on the connection super one-form $\tilde{A}$, to the zero-form gauge-fixing term $(\delta A=(\partial \cdot A))$ defined on the ordinary flat 2D Minkowskian spacetime manifold. This restriction is the analogue of horizontality condition w.r.t. $\tilde{\delta}$. Here the Hodge $\star$ duality operation is defined on the $(2+2)$-dimensional compact supermanifold. The basic superspace differentials transform under this operation as:

$$
\begin{array}{lcc}
\star\left(d x^{\mu}\right)=\varepsilon^{\mu \nu}\left(d x_{\nu}\right) & \star(d \theta)=(d \bar{\theta}) & \star(d \bar{\theta})=(d \theta) \\
\star(d \theta \wedge d \theta)=s^{\theta \theta} & \star(d \bar{\theta} \wedge d \bar{\theta})=s^{\bar{\theta} \bar{\theta}} & \star(d \theta \wedge d \bar{\theta})=s^{\theta \bar{\theta}} \\
\star\left(d x^{\mu} \wedge d x^{\nu}\right)=\varepsilon^{\mu \nu} & \star\left(d x^{\mu} \wedge d \theta\right)=\varepsilon^{\mu \theta} & \star\left(d x^{\mu} \wedge d \bar{\theta}\right)=\varepsilon^{\mu \bar{\theta}}
\end{array}
$$

where $\varepsilon^{\mu \theta(\bar{\theta})}=-\varepsilon^{\theta(\bar{\theta}) \mu}$ are anti-symmetric and $s^{\theta \bar{\theta}}=s^{\bar{\theta} \theta}$ etc. are symmetric. The expansion of the 1.h.s. of (4.2) defines a superscalar (zero-form) superfield

$$
\begin{aligned}
\tilde{\delta} \tilde{A} & =(\partial \cdot \Phi)+s^{\theta \theta}\left(\partial_{\theta} \eta\right)+s^{\bar{\theta} \bar{\theta}}\left(\partial_{\bar{\theta}} \bar{\eta}\right)+s^{\theta \bar{\theta}}\left(\partial_{\theta} \bar{\eta}+\partial_{\bar{\theta}} \eta\right) \\
& -\varepsilon^{\mu \theta}\left(\partial_{\mu} \eta+\varepsilon_{\mu \nu} \partial_{\theta} \Phi^{\nu}\right)-\varepsilon^{\mu \bar{\theta}}\left(\partial_{\mu} \bar{\eta}+\varepsilon_{\mu \nu} \partial_{\bar{\theta}} \Phi^{\nu}\right)
\end{aligned}
$$

\footnotetext{
$\ddagger \ddagger$ In any arbitrary flat Minowskian even $D$-dimensional ordinary spacetime manifolds, the ordinary dualexterior derivative $\delta$ is: $\delta=-* d *$. In general, an inner-product of a $n$-form in $D$-dimensional spacetime manifold leads to $\delta=(-1)^{D n+D+1} * d *$. Thus, for odd $D$, we have $\delta=(-1)^{n} * d *$ (see, e.g. Ref. [7]).
} 
and its subsequent equality with the ordinary gauge-fixing term in the r.h.s. (due to the analogue of the horizontality requirement) leads to

$$
\begin{array}{lll}
\partial_{\bar{\theta}} \bar{\eta}=0 & \rightarrow B=0 \quad \bar{s}=0 & \\
\partial_{\theta} \eta=0 & \rightarrow \bar{B}=0 \quad s=0 & \\
\partial_{\theta} \bar{\eta}=-\partial_{\bar{\theta}} \eta & \rightarrow \mathcal{B}+\overline{\mathcal{B}}=0 & \\
\partial_{\mu} \bar{\eta}=-\varepsilon_{\mu \nu} \partial_{\bar{\theta}} \Phi^{\nu} & \rightarrow R_{\mu}=-\varepsilon_{\mu \nu} \partial^{\nu} \bar{C} & S_{\mu}=+\varepsilon_{\mu \nu} \partial^{\nu} \overline{\mathcal{B}} \equiv-\varepsilon_{\mu \nu} \partial^{\nu} \mathcal{B} \\
\partial_{\mu} \eta=-\varepsilon_{\mu \nu} \partial_{\theta} \Phi^{\nu} & \rightarrow \bar{R}_{\mu}=-\varepsilon_{\mu \nu} \partial^{\nu} C & S_{\mu}=-\varepsilon_{\mu \nu} \partial^{\nu} \mathcal{B} .
\end{array}
$$

The above results also satisfy the following conditions:

$$
\partial \cdot \bar{R}=0 \quad \partial \cdot R=0 \quad \partial \cdot S=0 .
$$

It will be noticed that (4.4) can be directly obtained from (3.8) by exploiting the duality symmetry (2.9). In the language of the expansion of the superfields, we have obtained

$$
\begin{aligned}
\Phi_{\mu}(x, \theta, \bar{\theta}) & =A_{\mu}(x)-\theta \varepsilon_{\mu \nu} \partial^{\nu} C(x)-\bar{\theta} \varepsilon_{\mu \nu} \partial^{\nu} \bar{C}(x)-i \theta \bar{\theta} \varepsilon_{\mu \nu} \partial^{\nu} \mathcal{B}(x) \\
\eta(x, \theta, \bar{\theta}) & =C(x)+i \bar{\theta} \mathcal{B}(x) \\
\bar{\eta}(x, \theta, \bar{\theta}) & =\bar{C}(x)+i \theta \overline{\mathcal{B}}(x) \equiv \bar{C}(x)-i \theta \mathcal{B}(x) .
\end{aligned}
$$

This result can be further written in terms of the (anti-)dual BRST $s_{(a) d}$ symmetries (cf. eqns. (2.4) and (2.6)) obtained in Sec. 2, as follows

$$
\begin{aligned}
\Phi_{\mu}(x, \theta, \bar{\theta}) & =A_{\mu}(x)+\theta\left(s_{a d} A_{\mu}(x)\right)+\bar{\theta}\left(s_{d} A_{\mu}(x)\right)-\theta \bar{\theta}\left(s_{d} s_{a d} A_{\mu}(x)\right) \\
\eta(x, \theta, \bar{\theta}) & =C(x)+\theta\left(s_{a d} C(x)\right)-\bar{\theta}\left(s_{d} C(x)\right)+\theta \bar{\theta}\left(s_{d} s_{a d} C(x)\right) \\
\bar{\eta}(x, \theta, \bar{\theta}) & =\bar{C}(x)-\theta\left(s_{a d} \bar{C}(x)\right)+\bar{\theta}\left(s_{d} \bar{C}(x)\right)+\theta \bar{\theta}\left(s_{d} s_{a d} \bar{C}(x)\right) .
\end{aligned}
$$

These equations (4.5-4.7), finally, establish the origin of the existence of (anti-)dual BRST symmetries (discussed in Sec.2) in the framework of superfield formalism. It is evident that application of a single restriction (i.e., the analogue of horizontality condition) with the super co-exterior derivative (operating on $\tilde{A}$ ) leads to the derivation of two nilpotent (anti-) dual BRST symmetries for the 2D free Abelian gauge theory. It is worthwhile to compare and contrast transformations (3.11) vis-a-vis transformations (4.6) that are generated by (anti-)BRST- and (anti-)co-BRST charges $Q_{(a) b}$ and $Q_{(a) d}$, respectively. These are: (i) (anti-)BRST- and (anti-)co-BRST transformations correspond to translations along $\theta$ and $\bar{\theta}$ directions of the supermanifold for bosonic- and fermionic fields of the theory. (ii) For the bosonic field $A_{\mu}$, the above nilpotent charges generate translations along $\theta, \bar{\theta}$ and $\theta \bar{\theta}$ directions of the supermanifold. (iii) For the fermionic fields $C$ and $\bar{C}$, the translations are either along $\theta$ or $\bar{\theta}$ directions of the supermanifold. (iv) For the (anti-)ghost fields $(\bar{C}) C$, the (anti-)BRST charges generate translations along $(\bar{\theta}) \theta$ directions of the supermanifold. On the contrary, for the same fields, the (anti-)co-BRST charges generate translations along $(\theta) \bar{\theta}$ directions. (v) It is the mathematical power of $\tilde{d}$ (together with the Maurer-Cartan equation) that produces (anti-)BRST transformations. On the other hand, the derivation of the (anti-)co-BRST transformations depends entirely on the mathematical power of $\tilde{\delta}$. (vi) The mapping between (anti-)BRST- and (anti-)co-BRST charges on the one hand and 
the super cohomological operators $\tilde{d}$ and $\tilde{\delta}$ on the other hand, is: $Q_{(a) b} \Leftrightarrow \tilde{d}$ and $Q_{(a) d} \Leftrightarrow \tilde{\delta}$. (vii) The local conserved charges $Q_{(a) b}$ and $Q_{(a) d}$ are connected with the ordinary cohomological operators $d$ and $\delta$ through the mappings: $\left(Q_{b}, Q_{a d}\right) \Leftrightarrow d,\left(Q_{d}, Q_{a b}\right) \Leftrightarrow \delta[1,2,6]$.

\section{Discrete symmetry transformations in superfield formulation}

We have discussed the existence of a few discrete symmetries in Sec. 2. They are interesting in the sense that they enable us to gain some insights into various connections which exist among all BRST type symmetries that are present in the theory. In fact, it can be checked that the discrete transformations: $C \leftrightarrow \bar{C}, B \leftrightarrow-B,-\mathcal{B} \leftrightarrow-\mathcal{B}$ (that connect BRST- to anti-BRST and co-BRST- to anti-co-BRST symmetries) can be generalized vis-a-vis super expansion (3.2) in terms of superspace coordinates and component fields as

$$
C \leftrightarrow \bar{C}, \quad B \leftrightarrow-B, \quad \mathcal{B} \leftrightarrow-\mathcal{B}, \quad \theta \leftrightarrow \bar{\theta}, \quad R_{\mu} \leftrightarrow \bar{R}_{\mu}, \quad S_{\mu} \leftrightarrow-S_{\mu},
$$

under which the superfields transform as: $\Phi_{\mu}(x, \theta, \bar{\theta}) \leftrightarrow \Phi_{\mu}(x, \theta, \bar{\theta}), \eta(x, \theta, \bar{\theta}) \leftrightarrow \bar{\eta}(x, \theta, \bar{\theta})$. It will be noticed that for this discussion, we have taken $s(x)=\bar{s}(x)=0$ and $B+\bar{B}=$ $0, \mathcal{B}+\overline{\mathcal{B}}=0$ in expansion $(3.2)$, i.e.,

$$
\begin{aligned}
\Phi_{\mu}(x, \theta, \bar{\theta}) & =A_{\mu}(x)+\theta \bar{R}_{\mu}(x)+\bar{\theta} R_{\mu}(x)+i \theta \bar{\theta} S_{\mu}(x) \\
\eta(x, \theta, \bar{\theta}) & =C(x)-i \theta B(x)+i \bar{\theta} \mathcal{B}(x) \\
\bar{\eta}(x, \theta, \bar{\theta}) & =\bar{C}(x)-i \theta \mathcal{B}(x)+i \bar{\theta} B(x)
\end{aligned}
$$

due to the fact that these restrictions emerge when we show the existence of (anti-)BRSTand (anti-)co-BRST symmetries in the framework of superfield formulation. The Hodge decomposed versions for the $2 \mathrm{D}$ vector fields $\left(R_{\mu}, \bar{R}_{\mu}, S_{\mu}\right)$, consistent with transformations (5.1), can be written in terms of the ghost- and auxiliary fields as

$$
R_{\mu}=\partial_{\mu} C-\varepsilon_{\mu \nu} \partial^{\nu} \bar{C} \quad \bar{R}_{\mu}=\partial_{\mu} \bar{C}-\varepsilon_{\mu \nu} \partial^{\nu} C \quad S_{\mu}=\partial_{\mu} B-\varepsilon_{\mu \nu} \partial^{\nu} \mathcal{B}
$$

which obey $R_{\mu} \leftrightarrow \bar{R}_{\mu}, S_{\mu} \leftrightarrow-S_{\mu}$ under $C \leftrightarrow \bar{C}, B \leftrightarrow-B, \mathcal{B} \leftrightarrow-\mathcal{B}$. Now we wish to concentrate on the discrete symmetry transformations (2.9). This symmetry can be generalized in the framework of superfield formulation as

$$
\begin{array}{lllll}
C & \rightarrow \pm i \bar{C} & B \rightarrow \mp i \mathcal{B} & (\partial \cdot A) \rightarrow \pm i E & A_{\mu} \rightarrow A_{\mu} \\
\bar{C} \rightarrow \pm i C & \mathcal{B} \rightarrow \mp i B & E \rightarrow \pm i(\partial \cdot A) & S_{\mu} \rightarrow S_{\mu} \\
R_{\mu} \rightarrow-R_{\mu}, & \bar{R}_{\mu} \rightarrow-\bar{R}_{\mu}, & \theta \rightarrow-\theta \quad \bar{\theta} \rightarrow-\bar{\theta} & \partial_{\mu} \rightarrow \pm i \varepsilon_{\mu \nu} \partial^{\nu}
\end{array}
$$

under which the superfields transform as:

$$
\Phi_{\mu}(x, \theta, \bar{\theta}) \rightarrow \Phi_{\mu}(x, \theta, \bar{\theta}) \quad \eta(x, \theta, \bar{\theta}) \rightarrow \pm i \bar{\eta}(x, \theta, \bar{\theta}) \quad \bar{\eta}(x, \theta, \bar{\theta}) \rightarrow \pm i \eta(x, \theta, \bar{\theta})
$$

In the limit: $\theta \rightarrow 0, \bar{\theta} \rightarrow 0$, we get back transformations (2.9) from (5.3). It is interesting to point out that all the basic properties of the superfields $\eta^{2}=\bar{\eta}^{2}=0, \eta \bar{\eta}+\bar{\eta} \eta=0,\left[\Phi_{\mu}, \Phi_{\nu}\right]=$ 0 remain unchanged under all the discrete symmetry transformations (5.1) and (5.3). Now 
the Hodge decomposed versions for the $2 \mathrm{D}$ vectors $\left(R_{\mu}, \bar{R}_{\mu}, S_{\mu}\right)$, consistent with the discrete transformations (5.3), are

$$
R_{\mu}=\partial_{\mu} C+\varepsilon_{\mu \nu} \partial^{\nu} \bar{C} \quad \bar{R}_{\mu}=\partial_{\mu} \bar{C}+\varepsilon_{\mu \nu} \partial^{\nu} C \quad S_{\mu}=\partial_{\mu} B+\varepsilon_{\mu \nu} \partial^{\nu} \mathcal{B}
$$

which transform as: $R_{\mu} \rightarrow-R_{\mu}, \bar{R}_{\mu} \rightarrow-\bar{R}_{\mu}, S_{\mu} \rightarrow S_{\mu}$ under the transformations: $C \rightarrow$ $\pm i \bar{C}, \bar{C} \rightarrow \pm i C, B \rightarrow \mp i \mathcal{B}, \mathcal{B} \rightarrow \mp i B, \partial_{\mu} \rightarrow \pm i \varepsilon_{\mu \nu} \partial^{\nu}$. It should be pointed out here that in the derivation of the decompositions (5.2) and (5.5), the continuous symmetry transformations derived in (3.8), (3.11), (4.4) and (4.6) do play an important role.

It is important to note that the form of the Hodge decomposed versions (5.2) differs from the decompositions (5.5) only by a sign factor. Thus, the decompositions in (5.2) and (5.5) are orthogonal to each-other. This shows that the requirement of the consistency of the Hodge decompositions with the discrete symmetries of the theory forces two independent BRST-type nilpotent, continuous and covariant symmetries for the theory. These symmetries are none other than the (anti-)BRST- and the (anti-)co-BRST symmetries, we have discussed so far in the previous sections. It is very interesting to note that the Hodge decomposed versions for the $2 D$ fermionic vectors $R_{\mu}$ and $\bar{R}_{\mu}$ in (5.2) are also invariant under the discrete symmetry (duality) transformations (2.9). Thus, they can be called as "self-dual" as they transform to themselves under (2.9).

\section{Superfield approach to bosonic symmetry transformation}

It is useful for our discussions to calculate the operation of the ordinary Laplacian operator $\Delta=d \delta+\delta d$ on one-form $A=d x^{\mu} A_{\mu}$ defined on the 2D flat ordinary Minkowskian spacetime manifold. In fact, it turns out that the one-form $\Delta A$ can be expressed as

$$
\Delta\left(d x^{\mu} A_{\mu}\right)=d x^{\mu}\left[\partial_{\mu}(\partial \cdot A)-\varepsilon_{\mu \nu} \partial^{\nu} E\right] \equiv d x^{\mu} \square A_{\mu} .
$$

Thus, it is obvious that the operation of $\Delta$ on a form does not change the degree of the form. Equation (6.1) is going to be useful for the application of the analogue of the horizontality condition in the context of the operation of super Laplacian operator on the connection super one-form $\tilde{A}$. This condition can be succinctly expressed as the following equality

$$
\tilde{\Delta} \tilde{A}=(\tilde{d} \tilde{\delta}+\tilde{\delta} \tilde{d}) \tilde{A}=d x^{\mu} \square A_{\mu} .
$$

In other words, we expand the l.h.s. of the above equation in terms of the superspace differentials and set equal to zero all the components of $\tilde{\Delta} \tilde{A}$ that are found to be directed towards the Grassmannian directions $d \theta$ and $d \bar{\theta}$. In fact, (6.2) can be expressed as

$$
\begin{aligned}
& \tilde{d} \tilde{\delta} \tilde{A}=d x^{\rho} J_{\rho}+d \theta J_{\theta}+d \bar{\theta} J_{\bar{\theta}} \\
& \tilde{\delta} \tilde{d} \tilde{A}=d x^{\rho} H_{\rho}+d \theta H_{\theta}+d \bar{\theta} H_{\bar{\theta}}
\end{aligned}
$$


where the explicit expressions for the $J^{\prime} s$ are:

$$
\begin{aligned}
J_{\rho} & =\partial_{\rho}(\partial \cdot \Phi)-\varepsilon^{\lambda \bar{\theta}} \partial_{\rho}\left(\partial_{\lambda} \bar{\eta}+\varepsilon_{\lambda \mu} \partial_{\bar{\theta}} \Phi^{\mu}\right)+s^{\theta \theta} \partial_{\rho} \partial_{\theta} \eta \\
& +s^{\bar{\theta} \bar{\theta}} \partial_{\rho} \partial_{\bar{\theta}} \bar{\eta}-\varepsilon^{\lambda \theta} \partial_{\rho}\left(\partial_{\lambda} \eta+\varepsilon_{\lambda \mu} \partial_{\theta} \Phi^{\mu}\right)+s^{\theta \bar{\theta}} \partial_{\rho}\left(\partial_{\theta} \bar{\eta}+\partial_{\bar{\theta}} \eta\right) \\
J_{\theta} & =s^{\bar{\theta} \bar{\theta}} \partial_{\theta} \partial_{\bar{\theta}} \bar{\eta}+\partial_{\theta}(\partial \cdot \Phi)-\varepsilon^{\lambda \bar{\theta}}\left(\partial_{\theta} \partial_{\lambda} \bar{\eta}+\varepsilon_{\lambda \mu} \partial_{\theta} \partial_{\bar{\theta}} \Phi^{\mu}\right) \\
J_{\bar{\theta}} & =s^{\theta \theta} \partial_{\bar{\theta}} \partial_{\theta} \eta+\partial_{\bar{\theta}}(\partial \cdot \Phi)-\varepsilon^{\lambda \theta}\left(\partial_{\bar{\theta}} \partial_{\lambda} \eta+\varepsilon_{\lambda \mu} \partial_{\bar{\theta}} \partial_{\theta} \Phi^{\mu}\right)
\end{aligned}
$$

and that of $H^{\prime} s$ are:

$$
\begin{aligned}
H_{\rho} & =\varepsilon_{\rho \sigma} \partial^{\sigma}\left(\varepsilon^{\mu \nu} \partial_{\mu} \Phi_{\nu}\right)+\varepsilon^{\lambda \bar{\theta}} \varepsilon_{\rho \sigma} \partial^{\sigma}\left(\partial_{\lambda} \eta-\partial_{\bar{\theta}} \Phi_{\lambda}\right)-s^{\theta \theta} \varepsilon_{\rho \sigma} \partial^{\sigma} \partial_{\theta} \bar{\eta} \\
& -s^{\bar{\theta} \bar{\theta}} \varepsilon_{\rho \sigma} \partial^{\sigma} \partial_{\bar{\theta}} \eta+\varepsilon^{\lambda \theta} \varepsilon_{\rho \sigma} \partial^{\sigma}\left(\partial_{\lambda} \bar{\eta}-\partial_{\theta} \Phi_{\lambda}\right)-s^{\theta \bar{\theta}} \varepsilon_{\rho \sigma} \partial^{\sigma}\left(\partial_{\bar{\theta}} \bar{\eta}+\partial_{\theta} \eta\right) \\
H_{\theta} & =s^{\theta \theta} \partial_{\bar{\theta}} \partial_{\theta} \bar{\eta}-\partial_{\bar{\theta}}\left(\varepsilon^{\mu \nu} \partial_{\mu} \Phi_{\nu}\right)-\varepsilon^{\lambda \theta}\left(\partial_{\bar{\theta}} \partial_{\lambda} \bar{\eta}-\partial_{\bar{\theta}} \partial_{\theta} \Phi_{\lambda}\right) \\
H_{\bar{\theta}} & =s^{\bar{\theta} \bar{\theta}} \partial_{\theta} \partial_{\bar{\theta}} \eta-\partial_{\theta}\left(\varepsilon^{\mu \nu} \partial_{\mu} \Phi_{\nu}\right)-\varepsilon^{\lambda \bar{\theta}}\left(\partial_{\theta} \partial_{\lambda} \eta-\partial_{\theta} \partial_{\bar{\theta}} \Phi_{\lambda}\right) .
\end{aligned}
$$

In the computations of (6.4) and (6.5), we have exploited the anti-symmetry- and symmetry properties of $\varepsilon^{\mu \theta(\bar{\theta})}$ and $s^{\theta \bar{\theta}}$ respectively. We have also used: $\left(\partial_{\theta}\right)^{2}=0,\left(\partial_{\bar{\theta}}\right)^{2}=0$, etc.

Now we impose the analogue of the horizontality condition. In fact, the condition $d \theta\left(J_{\theta}+H_{\theta}\right)=0$ leads to setting the coefficients of $s^{\theta \theta}, s^{\bar{\theta} \bar{\theta}}, \varepsilon^{\mu \theta}, \varepsilon^{\mu \bar{\theta}}$ equal to zero. These are

$$
\bar{s}=0 \quad \partial_{\theta}(\partial \cdot \Phi)=\partial_{\bar{\theta}}\left(\varepsilon^{\mu \nu} \partial_{\mu} \Phi_{\nu}\right) \quad \partial_{\theta}\left(\partial_{\mu} \bar{\eta}+\varepsilon_{\mu \nu} \partial_{\bar{\theta}} \Phi^{\nu}\right)=0 \quad \partial_{\bar{\theta}}\left(\partial_{\mu} \bar{\eta}-\partial_{\theta} \Phi_{\mu}\right)=0 .
$$

In the language of the component fields of the expansion (3.2), these conditions lead to

$$
\partial \cdot S=0 \quad \varepsilon^{\mu \nu} \partial_{\mu} S_{\nu}=0 \quad \partial \cdot \bar{R}=\varepsilon^{\mu \nu} \partial_{\mu} R_{\nu} \quad S_{\mu}=\partial_{\mu} B \quad S_{\mu}=\varepsilon_{\mu \nu} \partial^{\nu} \overline{\mathcal{B}}
$$

which imply the results that are listed below

$$
\bar{R}_{\mu}=\varepsilon_{\mu \nu} R^{\nu} \quad \square S_{\mu}=0 \quad S_{\mu}=\frac{1}{2}\left[\partial_{\mu} B+\varepsilon_{\mu \nu} \partial^{\nu} \overline{\mathcal{B}}\right] \quad \partial_{\mu} B-\varepsilon_{\mu \nu} \partial^{\nu} \overline{\mathcal{B}}=0 .
$$

It will be noticed that conditions $\partial \cdot S=0$ and $\varepsilon^{\mu \nu} \partial_{\mu} S_{\nu}=0$ imply $\square S_{\mu}=0$. We also obtain $\square B=0, \square \overline{\mathcal{B}}=0$. Similarly, the imposition of the constraint $d \bar{\theta}\left(J_{\bar{\theta}}+H_{\bar{\theta}}\right)=0$, leads to

$$
s=0 \quad \partial_{\bar{\theta}}(\partial \cdot \Phi)=\partial_{\theta}\left(\varepsilon^{\mu \nu} \partial_{\mu} \Phi_{\nu}\right) \quad \partial_{\bar{\theta}}\left(\partial_{\mu} \eta+\varepsilon_{\mu \nu} \partial_{\theta} \Phi^{\nu}\right)=0 \quad \partial_{\theta}\left(\partial_{\mu} \eta-\partial_{\bar{\theta}} \Phi_{\mu}\right)=0 .
$$

The above equations, vis-a-vis expansions in (3.2), lead to

$$
\partial \cdot S=0 \quad \varepsilon^{\mu \nu} \partial_{\mu} S_{\nu}=0 \quad \partial \cdot R=\varepsilon^{\mu \nu} \partial_{\mu} \bar{R}_{\nu} \quad S_{\mu}=-\partial_{\mu} \bar{B} \quad S_{\mu}=-\varepsilon_{\mu \nu} \partial^{\nu} \mathcal{B} .
$$

These equations imply the following results

$$
R_{\mu}=\varepsilon_{\mu \nu} \bar{R}^{\nu} \quad \square S_{\mu}=0 \quad S_{\mu}=-\frac{1}{2}\left[\partial_{\mu} \bar{B}+\varepsilon_{\mu \nu} \partial^{\nu} \mathcal{B}\right] \quad \partial_{\mu} \bar{B}-\varepsilon_{\mu \nu} \partial^{\nu} \mathcal{B}=0
$$

which, in turn, lead to $\square \bar{B}=0, \square \mathcal{B}=0$. It will be interesting to point out that the conditions $R_{\mu}=\varepsilon_{\mu \nu} \bar{R}^{\nu}, \bar{R}_{\mu}=\varepsilon_{\mu \nu} R^{\nu}$ are satisfied for both the decompositions (5.2) as well as (5.5). The final requirement due to the analogue of horizontality condition is

$$
d x^{\rho}\left(J_{\rho}+H_{\rho}\right)=d x^{\rho} \square A_{\rho} .
$$


A close look at the expressions for $J_{\rho}, H_{\rho}$ and (6.12) shows that

$$
d x^{\rho}\left[\partial_{\rho}(\partial \cdot \Phi)+\varepsilon_{\rho \sigma} \partial^{\sigma}\left(\varepsilon^{\mu \nu} \partial_{\mu} \Phi_{\nu}\right)\right]=d x^{\rho} \square A_{\rho}
$$

where the l.h.s. reduces to $\left(d x^{\rho} \square \Phi_{\rho}\right)$. This leads to the following restrictions on $R_{\mu}, \bar{R}_{\mu}, S_{\mu}$ :

$$
\square R_{\mu}=0 \quad \square \bar{R}_{\mu}=0 \quad \square S_{\mu}=0 .
$$

Hereafter, in the rest of computations, we set $s(x)=0, \bar{s}(x)=0$ in the expansion (3.2) (cf.(6.6),(6.9)). Now setting the coefficients of $\left(d x^{\rho} s^{\theta \theta}\right)$ and $\left(d x^{\rho} s^{\bar{\theta} \bar{\theta}}\right)$ equal to zero leads to:

$$
\partial_{\theta}\left(\partial_{\rho} \eta-\varepsilon_{\rho \sigma} \partial^{\sigma} \bar{\eta}\right)=0 \quad \partial_{\bar{\theta}}\left(\partial_{\rho} \bar{\eta}-\varepsilon_{\rho \sigma} \partial^{\sigma} \eta\right)=0 .
$$

The above equations produce the following two restrictions:

$$
\partial_{\rho} B-\varepsilon_{\rho \sigma} \partial^{\sigma} \mathcal{B}=0 \quad \partial_{\rho} \bar{B}-\varepsilon_{\rho \sigma} \partial^{\sigma} \overline{\mathcal{B}}=0 .
$$

It will be noted that these restrictions automatically satisfy the conditions that emerge due to setting the coefficient of $\left(d x^{\rho} s^{\theta \bar{\theta}}\right)$ equal to zero. A comparison between (6.8) and (6.11) on the one hand and (6.16) on the other hand, produces the conditions: $B=\bar{B}, \mathcal{B}=$ $\overline{\mathcal{B}}, \partial_{\rho} B-\varepsilon_{\rho \sigma} \partial^{\sigma} \mathcal{B}=0$. However, with these equalities, it is clear that equations (6.8) and (6.11) lead to: $S_{\mu}=0, \partial_{\rho} B+\varepsilon_{\rho \sigma} \partial^{\sigma} \mathcal{B}=0$. Thus, we have got the orthogonal combinations of derivatives on $B$ and $\mathcal{B}$ that are equal to zero. Ultimately, these imply that we can choose: $B=\bar{B}=\mathcal{B}=\overline{\mathcal{B}}=0$. At this stage, it is worthwhile to emphasize that, as the operator equations, these conditions are not unexpected. In fact, it has been shown that the choice of the physical state as the harmonic state in the Hodge decomposition allows one to require that: $Q_{a(b)} \mid$ phys $>=0, Q_{a(d)} \mid$ phys $>=0$ which imply that $B \mid$ phys $>=0, \mathcal{B} \mid$ phys $>=0$ (cf.(7.1)) $[1,2,6]$. There is one more subtle point that has to be stressed here. Even though, $S_{\mu}=0$ in terms of $B$ and $\mathcal{B}$, there is another choice of $S_{\mu}$ that remains invariant under the discrete symmetry transformations (2.9). For instance, $S_{\mu}=-\varepsilon_{\mu \nu} \square A^{\nu}$ remains invariant under the duality transformations (2.9) because $A_{\mu} \rightarrow A_{\mu}$ and $\square \rightarrow \square$ under $\partial_{\mu} \rightarrow \pm i \varepsilon_{\mu \nu} \partial^{\nu}$. Furthermore, the conditions $\partial \cdot S=0$ and $\varepsilon^{\mu \nu} \partial_{\mu} S_{\nu}=0$ are automatically satisfied because $\square(\partial \cdot A)=0$ and $\square E=0$ due to the equations of motion (with $B=-(\partial \cdot A), \mathcal{B}=E$ ). Thus, we shall persist with $S_{\mu}$ in our further discussions.

For the rest of our computations, we shall set $s(x)=\bar{s}(x)=0$ and $B(x)=\bar{B}(x)=$ $\mathcal{B}(x)=\overline{\mathcal{B}}(x)=0$ in our superfield expansion (3.2). With these insertions in (3.2), it is obvious now that setting of the coefficients of $\left(d x^{\rho} \varepsilon^{\lambda \theta}\right)$ and $\left(d x^{\rho} \varepsilon^{\lambda \bar{\theta}}\right)$ equal to zero, leads to

$$
\begin{aligned}
& \varepsilon_{\rho \sigma} \partial^{\sigma}\left(\partial_{\lambda} \bar{\eta}-\partial_{\theta} \Phi_{\lambda}\right)-\partial_{\rho}\left(\partial_{\lambda} \eta+\varepsilon_{\lambda \mu} \partial_{\theta} \Phi^{\mu}\right)=0 \\
& \varepsilon_{\rho \sigma} \partial^{\sigma}\left(\partial_{\lambda} \eta-\partial_{\bar{\theta}} \Phi_{\lambda}\right)-\partial_{\rho}\left(\partial_{\lambda} \bar{\eta}+\varepsilon_{\lambda \mu} \partial_{\bar{\theta}} \Phi^{\mu}\right)=0 .
\end{aligned}
$$

In terms of the component fields of (3.2), the above equations produce the following conditions:

$$
\begin{aligned}
& \partial_{\lambda}\left(\varepsilon_{\rho \sigma} \partial^{\sigma} \bar{C}-\partial_{\rho} C\right)=\partial_{\rho} R_{\lambda}+\varepsilon_{\rho \sigma} \partial^{\sigma} \bar{R}_{\lambda} \\
& \partial_{\lambda}\left(\varepsilon_{\rho \sigma} \partial^{\sigma} C-\partial_{\rho} \bar{C}\right) \\
& \varepsilon_{\rho \sigma} \partial^{\sigma} S_{\lambda}+\partial_{\rho}\left(\varepsilon_{\lambda \mu} S^{\mu}\right)=0
\end{aligned}
$$


where $R_{\mu}=\varepsilon_{\mu \nu} \bar{R}^{\nu}, \bar{R}_{\mu}=\varepsilon_{\mu \nu} R^{\nu}$. The last equation, contracted with $\partial_{\lambda}$, is automatically satisfied due to $(\partial \cdot S)=0$ and $\varepsilon^{\mu \nu} \partial_{\mu} S_{\nu}=0$. Moreover, the top equation can be re-expressed, in terms of the component fields, as

$$
-\partial_{\lambda}\left(\varepsilon_{\rho \sigma} \partial^{\sigma} C-\partial_{\rho} \bar{C}\right)=\partial_{\rho} \bar{R}_{\lambda}+\varepsilon_{\rho \sigma} \partial^{\sigma} R_{\lambda} .
$$

Comparison of the central equation of (6.18) with the above equation, yields

$$
\partial_{\lambda}\left(\varepsilon_{\rho \sigma} \partial^{\sigma} C-\partial_{\rho} \bar{C}\right)=0 \quad \partial_{\rho} \bar{R}_{\lambda}+\varepsilon_{\rho \sigma} \partial^{\sigma} R_{\lambda}=0 .
$$

One of the very interesting solutions for the above conditions is $\bar{R}_{\rho}=\partial_{\rho} \bar{C}-\varepsilon_{\rho \sigma} \partial^{\sigma} C=0$ which is equivalent to setting the Hodge decomposed version of equation (5.2) equal to zero. This, in turn, implies that the other $2 \mathrm{D}$ fermionic vector $R_{\rho}=\partial_{\rho} C-\varepsilon_{\rho \sigma} \partial^{\sigma} \bar{C}$ is also equal to zero. Thus, ultimately, we have obtained the following solution from the analogue of the horizontality condition w.r.t. the super Laplacian operator $\tilde{\Delta}$

$$
\begin{aligned}
& R_{\mu}(x)=\bar{R}_{\mu}(x)=0 \quad B(x)=\bar{B}(x)=\mathcal{B}(x)=\overline{\mathcal{B}}(x)=0 \\
& S_{\mu}(x)=-\varepsilon_{\mu \nu} \square A^{\nu}(x) \equiv\left(\tilde{s}_{w} A_{\mu}(x)\right) \quad s(x)=\bar{s}(x)=0 .
\end{aligned}
$$

The superfield expansion (3.2), with the above inputs, bears an appearance as

$$
\Phi_{\mu}(x, \theta, \bar{\theta})=A_{\mu}(x)+i \theta \bar{\theta}\left(\tilde{s}_{w} A_{\mu}(x)\right) \quad \eta(x, \theta, \bar{\theta})=C(x) \quad \bar{\eta}(x, \theta, \bar{\theta})=\bar{C}(x) .
$$

This shows that the ghost fields $C(x)$ and $\bar{C}(x)$ do not transform at all under $\tilde{s}_{w}$ and gauge field $A_{\mu}(x)$ transforms to its own equation of motion along the bosonic $(\theta \bar{\theta})$ direction of the expansion. This establishes the fact that symmetry transformations $\tilde{s}_{w} C=0, \tilde{s}_{w} \bar{C}=$ $0, \tilde{s}_{w} A_{\mu}=-\varepsilon_{\mu \nu} \square A^{\nu}$, which are the analogues of (2.7) (with $\mathcal{B}=E, B=-(\partial \cdot A)$ ), are generated by a conserved bosonic charge $Q_{w}$ (cf.(7.1)) which owes its origin to the super Laplacian operator $\tilde{\Delta}$. Geometrically, the operator $Q_{w}$ turns out to be the translation generator along a bosonic direction that is equivalent to a couple of successive translations along $\theta$ and $\bar{\theta}$ directions (i.e. $\operatorname{Lim}_{\theta, \bar{\theta} \rightarrow 0} \frac{\partial}{\partial \theta} \frac{\partial}{\partial \theta} \Phi_{\mu}(x, \theta, \bar{\theta})=\left[A_{\mu}, Q_{w}\right]$ ).

\section{Conclusions}

In the present investigation, we have exploited the mathematical power of super de Rham cohomology operators $(\tilde{d}, \tilde{\delta}, \tilde{\Delta})$ of differential geometry and a generalized version of the socalled horizontality condition [13-18] for the geometrical interpretation of the local Noether conserved charges $\left(Q_{(a) b}, Q_{(a) d}, Q_{w}\right)$ as the generators of translations along the Grassmannian (odd)- and bosonic (even) directions of a compact $(2+2)$-dimensional supermanifold. The explicit local expressions for all these conserved Noether charges, together with the ghost charge $Q_{g}$, are $[1,2,6]$

$$
\begin{aligned}
Q_{b} & =\int d x[B \dot{C}-\dot{B} C] & & Q_{a b}=\int d x[B \dot{\bar{C}}-\dot{B} \bar{C}] \\
Q_{d} & =\int d x[\mathcal{B} \overline{\bar{C}}-\dot{\mathcal{B}} \bar{C}] & & Q_{a d}=\int d x[\mathcal{B} \dot{C}-\dot{\mathcal{B} C}] \\
Q_{w} & =\int d x[B \dot{\mathcal{B}}-\dot{B} \mathcal{B}] & & Q_{g}=-i \int d x[C \dot{\bar{C}}+\bar{C} \dot{C}]
\end{aligned}
$$


which obey the following algebra $[1,2,6]$

$$
\begin{array}{ll}
Q_{b}^{2}=Q_{d}^{2}=Q_{a b}^{2}=Q_{a d}^{2}=0 & \left\{Q_{b}, Q_{a b}\right\}=\left\{Q_{d} Q_{a d}\right\}=0 \\
i\left[Q_{g}, Q_{b(a d)}\right]=+Q_{b(a d)} & i\left[Q_{g}, Q_{d(a b)}\right]=-Q_{d(a b)} \\
Q_{w}=\left\{Q_{b}, Q_{d}\right\}=\left\{Q_{a d}, Q_{a b}\right\} & {\left[Q_{w}, Q_{k}\right]=0 \quad k=a, a b, d, a d, g}
\end{array}
$$

if we exploit the canonical (anti-)commutation relations for the Lagrangian density (2.2). The above algebra is reminiscent of the algebra obeyed by the de Rham cohomology operators of differential geometry (see,e.g.,Sec.1) and it remains form-invariant under the discrete duality transformations (2.9). The degree of a given differential form is equivalent to the ghost number of a quantum state in the BRST formalism as (1.1) and (7.2) imply

$$
\begin{aligned}
& i Q_{g} Q_{b(a d)}\left|\Psi>_{n}=(n+1) Q_{b(a d)}\right| \Psi>_{n} \\
& i Q_{g} Q_{d(a b)}\left|\Psi>_{n}=(n-1) Q_{d(a b)}\right| \Psi>_{n} \\
& i Q_{g} Q_{w}\left|\Psi>_{n}=n Q_{w}\right| \Psi>_{n} .
\end{aligned}
$$

The above equations re-establish the identification of conserved local Noether charges with the de Rham cohomology operators of differential geometry because as operators $d(\delta)$ increase(reduce) the degree of a form by one so do the local charges $Q_{b(a d)}\left(Q_{d(a b)}\right)$ to a state with a certain ghost number. Thus, one has the following mappings: $d \Leftrightarrow\left(Q_{b}, Q_{a d}\right), \delta \Leftrightarrow$ $\left(Q_{d}, Q_{a b}\right), \Delta \Leftrightarrow Q_{w}=\left\{Q_{(a) b}, Q_{(a) d}\right\}$. With these identifications, the HDT and the BRST cohomology can be defined comprehensively in the quantum Hilbert space of states $[1,2,6,11]$. The existence of some new discrete symmetries in the superfield formulation leads to some key insights which enable us to obtain the Hodge decomposition for the 2D odd vectors of the superfield expansion (3.2). The interplay between discrete and continuous symmetries of the theory (together with the idea of the HDT) provides the origin for the existence of (anti-)BRST- and (anti-) co-BRST symmetries. The requirement of the self-duality invariance of the theory under (2.9) provides a logical reason for the choice of the Hodge decomposed version (5.2) over its counterpart (5.5) in the solution (6.20). In fact, this solution finally leads to the derivation of the bosonic symmetry where $R_{\mu}=\bar{R}_{\mu}=0$. The self-duality invariance also provides a very convincing guess for the correct Hodge decomposed version of $2 \mathrm{D}$ bosonic vectors: $S_{\mu}=\partial_{\mu} B+\varepsilon_{\mu \nu} \partial^{\nu} \mathcal{B}$ and $A_{\mu}=\partial_{\mu} \lambda+\varepsilon_{\mu \nu} \partial^{\nu} \kappa$ where $\lambda$ and $\kappa$ are some scalar fields (see, e.g., Ref. [2] for details).

In our earlier works $[6,1,2]$, it has been demonstrated that $2 \mathrm{D}$ free Abelian $U(1)$ gauge theory in the flat Minkowski spacetime is a new type of topological field theory (TFT) [23] as the BRST- and dual BRST symmetries gauge out the propagating degrees of freedom of the $2 \mathrm{D}$ photon. This new theory captures together some of the key features of Wittenas well as Schwarz type TFTs [24,25]. In fact, the form of the quantum action for this theory bears the appearance of a Witten type theory but the symmetries are local gauge type which resemble very much like symmetries of the Schwarz type TFT. The topological nature of this theory has been shown to be encoded in the vanishing of the Laplacian operator $Q_{w} \rightarrow 0$ when the on-shell condition for photon (i.e. $\partial_{\mu} B+\varepsilon_{\mu \nu} \partial^{\nu} \mathcal{B}=0$ ) is utilized $[1,2,6]$. In fact, under this condition, all the transformations in (2.7) become trivially zero. 
In the framework of superfield formulation, this fact is encoded in the vanishing of the 2D fermionic vectors $R_{\mu}$ and $\bar{R}_{\mu}$ and bosonic $2 \mathrm{D}$ vector $S_{\mu}$ turns out to be equal to $-\varepsilon_{\mu \nu} \square A^{\nu}$ only. Thus, on the on-shell (i.e. $\square A_{\mu}=0$ ), even $S_{\mu}$ turns out to be zero. The topological nature of the $2 \mathrm{D}$ free $U(1)$ gauge theory is captured in the horizontality condition of the superfield formulation. In fact, it can be seen that the restriction $\tilde{\Delta} \tilde{A}=\Delta A\left(\equiv d x^{\rho} \square A_{\rho}\right)$ vanishes on the on-shell $\left(\square A_{\mu}=0\right)$ of the $2 \mathrm{D}$ photon which, ultimately, implies $Q_{w} \rightarrow 0$. In a recent paper [26], a very direct, simple and straightforward method has been adopted to capture some of the topological features of our present theory where an explicit presence of the superfields $\Phi_{\mu}(x, \theta, \bar{\theta}), \eta(x, \theta, \bar{\theta})$ and $\bar{\eta}(x, \theta, \bar{\theta})$ has been invoked.

It is important to point out that, in the superfield formulation on the $(2+2)$-dimensional supermanifold, one can define forms that have greater degree than the spacetime dimension of the supermanifold. However, the generalized versions of the horizontality condition w.r.t. all the super cohomological operators (which we have exploited in our present discussion) turn out to be quite handy to resolve this anomaly. It would be nice to take an explicit example of a supersymmetric theory and work out all the details of the HDT for this theory in the quantum Hilbert space of states. At the moment, we are looking into this problem and our results will be reported in our future publications.

An interesting generalization of our present superfield formulation for the free 2D Abelian gauge theory to self-interacting 2D non-Abelian gauge theory has already been studied in Ref. [27]. It would be very interesting endeavour to look for the field theoretical models for the Hodge theory where there is an interaction between matter- and gauge fields. Some steps in this direction have already been taken [3,4] for the interacting 2D Abelian gauge theory. It has been shown in these works that, as (anti-)BRST symmetries are connected with the local gauge symmetries on gauge- and Dirac fields, similarly the (anti-)co-BRST symmetries on the gauge fields are connected with the analogue of chiral symmetries on the Dirac fields. The local Noether charges, corresponding to these symmetries, turn out to be the analogues of cohomolgical operators $d$ and $\delta$. We firmly believe that a thorough study of this interacting theory with the Wess-Zumino term would shed light on the consistency and unitarity of the 2D anomalous gauge theory (see, e.g., Refs. $[28,29]$ and references therein) in the framework of BRST cohomology and HDT. It would be also interesting to generalize our results to the free 4D two-form Abelian gauge theory

where the existence of analogues of the local conserved charges $Q_{(a) b}, Q_{(a) d}, Q_{w}$ has been established. These are some of the issues that are under investigation [30].

\section{Acknowledgements}

The enlightening and thought-provoking comments by the adjudicator on some of the key issues of this paper are gratefully acknowledged. Thanks are also due to A. Lahiri for his interest in this work. 


\section{References}

[1] Malik R P 2000 J. Phys. A: Math Gen 332437

(Malik R P 1999 Preprint hep-th/9902146)

[2] Malik R P 2000 Int. J. Mod. Phys. A 151685

(Malik R P 1998 Preprint hep-th/9808040)

[3] Malik R P 2000 Mod. Phys. Lett. A 152079

(Malik R P 2000 Preprint hep-th/0003128)

[4] Malik R P 2001 Mod. Phys. Lett. A 16477

(Malik R P 1997 Preprint hep-th/9711056)

[5] Malik R P 1999 Mod. Phys. Lett. A 141937

(Malik R P 1999 Preprint hep-th/9903121)

[6] Malik R P 2001 J. Phys. A: Math Gen 344167

(Malik R P 2000 Preprint hep-th/0012085)

[7] Eguchi T, Gilkey P B and Hanson A J 1980 Phys. Rep. 66213

[8] Mukhi S and Mukunda N 1990 Introduction to Topology, Differential Geometry and Group Theory for Physicists (New Delhi: Wiley)

[9] Nishijima K 1988 Prog. Theo. Phys. 80897

van Holten J W 1990 Phys. Rev. Lett. 642867

Aratyn H 1990 J. Math. Phys. 311240

[10] Fülöp G and Marnelius R 1995 Nucl. Phys. B 456442

Kalau W and van Holten J W 1991 Nucl. Phys. B 261233

[11] Harikumar E, Malik R P and Sivakumar M 2000 J. Phys. A: Math Gen 337149

(Harikumar E, Malik R P and Sivakumar M 2000 Preprint hep-th/0004145)

[12] Malik R P 2001 Phys. Lett. B 521409

(Malik R P 2001 Preprint hep-th/0108105)

[13] Thierry-Mieg J 1980 J. Math. Phys. 212834

Thierry-Mieg J 1980 Nuovo Cim. 56 A 396

[14] Quiros M, De Urries F J, Hoyos J, Mazou M L and Rodrigues E 1981 J. Math. Phys. 22767

[15] Bonora L and Tonin M 1981 Phys. Lett. B 9848

Bonora L, Pasti P and Tonin M 1981 Nuovo Cim. 63 A 353

[16] Baulieu L and Thierry-Mieg J 1982 Nucl. Phys. B 197477

Baulieu L and Thierry-Mieg J 1982 Nucl. Phys. B 228259

Alvarez-Gaumé L and Baulieu L 1983 Nucl. Phys. B 212255

[17] Hwang D S and Lee C -Y 1997 J. Math. Phys. 3830

[18] Nakanishi N and Ojima I 1990 Covariant Operator Formalism of Gauge Theories and Quantum Gravity (Singapore: World Scientific)

[19] Nishijima K 1986 Progress in Quantum Field Theory ed H Ezawa and S Kamefuchi (Amsterdam: North- Holland ) 
[20] Henneaux M and Teitelboim C 1992 Quantization of Gauge Systems (Princeton, NJ: Princeton University Press)

[21] Weinberg S 1996 The Quantum Theory of Fields: Modern Applications, Vol 2 (Cambridge: Cambridge University Press)

[22] Wess J and Bagger J 1991 Supersymmetry and Supergravity (2nd Edition) (Princeton, NJ: Princeton University Press)

[23] Birmingham D, Blau M, Rakowski M and Thompson G 1991 Phys. Rep. 209129

[24] Witten E 1989 Commun. Math. Phys. 121351

[25] Schwarz A S 1978 Lett. Math. Phys. 2217

[26] Malik R P 2001 Topological aspects of Abelian gauge theory in superfield formalism (Malik R P 2001 Preprint hep-th/0112260)

[27] Malik R P 2002 Mod. Phys. Lett. A 17185

(Malik R P 2001 Preprint hep-th/0111253)

[28] Jackiw R and Rajaraman R 1985 Phys. Rev. Lett. 541219

[29] Malik R P 1988 Phys. Lett. B 212445

[30] Malik R P 2002 in preparation 